\title{
Comparative Evaluation of Antimicrobial Efficacy of Mineral Trioxide Aggregate, Biodentine, EndoSequence against Bacterial Strains of Enterococcus faecalis- An In-Vitro Study
}

Rajlaxmi Kadam¹, Shashikiran², Pooja Mapara ${ }^{3}$, Namrata Gaonkar4, Sachin Gugawad5, Savita Hadakar6, Swapnil Taur ${ }^{7}$

\begin{abstract}
${ }^{1}$ Department of Paedodontics and Preventive Dentistry, School of Dental Sciences, Krishna Institute of Medical Sciences Deemed University, Karad, Maharashtra, India. ${ }^{2}$ Department of Paedodontics and Preventive Dentistry, School of Dental Sciences, Krishna Institute of Medical Sciences Deemed University, Karad, Maharashtra, India. ${ }^{3}$ Department of Paedodontics and Preventive Dentistry, School of Dental Sciences, Krishna Institute of Medical Sciences Deemed University, Karad, Maharashtra, India. ${ }^{4}$ Department of Paedodontics and Preventive Dentistry, School of Dental Sciences, Krishna Institute of Medical Sciences Deemed University, Karad, Maharashtra, India. ${ }^{5}$ Department of Paedodontics and Preventive Dentistry, School of Dental Sciences, Krishna Institute of Medical Sciences Deemed University, Karad, Maharashtra, India. ${ }^{6}$ Department of Paedodontics and Preventive Dentistry, School of Dental Sciences, Krishna Institute of Medical Sciences Deemed University, Karad,

Maharashtra, India. ${ }^{7}$ Department of Paedodontics and Preventive Dentistry, School of Dental Sciences, Krishna Institute of Medical Sciences Deemed University, Karad, Maharashtra, India.
\end{abstract}

\section{ABSTRACT}

\section{BACKGROUND}

Microorganisms are the common aetiological factors in initiation as well as perpetuation of periapical and pulpal diseases. Microbial leakage is a cardinal aetiology behind endodontic treatment failure. The success of root canal treatment is greatly influenced by antimicrobial potential of the dental material used in endodontic procedures which plays an important role in creating a hermetic seal to prevent egress of bacteria into root canal from periapical region.

\section{METHODS}

The three cements MTA, Biodentine and EndoSequence were made into pellets to test their antimicrobial action on the growth of Enterococcus faecalis (ATCC 29212); method used was agar diffusion test.

\section{RESULTS}

At 24-hour study interval, EndoSequence showed the greatest zone of inhibition of $10.6 \mathrm{~mm}$ against Enterococcus faecalis (ATCC 29212) compared to Biodentine with zone of inhibition of $5.5 \mathrm{~mm}$ and MTA with the zone of inhibition of $5 \mathrm{~mm}$. EndoSequence showed highest antimicrobial efficacy at 36-hours and 72-hours study intervals by mean inhibition zone of $11 \mathrm{~mm}$ against Enterococcus faecalis compared to that of Biodentine $(6 \mathrm{~mm})$ and MTA $(5.6 \mathrm{~mm})$ respectively with a p value of less than 0.05 which is highly significant. The antimicrobial activity of EndoSequence was very strong compared to Biodentine and MTA against Enterococcus faecalis (ATCC 29212).

\section{CONCLUSIONS}

Microorganisms play pivotal role in the initiation as well as perpetuation of periapical and pulpal diseases. Microbial leakage is a cardinal aetiology behind endodontic treatment failure. The success of the root canal treatment is greatly influenced by antimicrobial potential of the dental material used in endodontic procedures which plays an important role in creating a hermetic seal to prevent egress of bacteria into root canal from periapical region.

\section{KEY WORDS}

Mineral Trioxide Aggregate, Biodentine, EndoSequence, Antimicrobial Efficacy, Enterococcus faecalis

\author{
Corresponding Author: \\ Dr. Shashikiran N. D. \\ Dean and HOD, \\ Department of Paedodontics and \\ Preventive Dentistry, School of Dental \\ Sciences, Krishna Institute of Medical \\ Sciences, Deemed University, Karad, \\ Satara-415110, Maharashtra, India. \\ E-mail: drndshashikiran@gmail.com
}

DOI: $10.14260 /$ jemds/2020/433

How to Cite This Article:

Kadam R, Shashikiran ND, Mapara P, et al. Comparative evaluation of antimicrobial efficacy of mineral trioxide aggregate, biodentine, endosequence against bacterial strains of Enterococcus faecalis- an in-vitro study. J. Evolution Med. Dent. Sci. 2020;9(28):1988-1991, DOI: 10.14260/jemds/2020/433

Submission 11-04-2020,

Peer Review 05-06-2020,

Acceptance 11-06-2020,

Published 13-07-2020.

Copyright (C) 2020 JEMDS. This is an open access article distributed under Creative Commons Attribution License [Attribution 4.0 International (CC BY 4.0)] 


\section{BACKGROUND}

Microorganisms are the common aetiological factors in initiation as well as perpetuation of periapical and pulpal diseases.[1] Microbial leakage is a cardinal aetiology behind endodontic treatment failure.[2] The success of the root canal treatment is amalgamation of careful diagnosis, efficiently performed chemo-mechanical preparation of root canals, and compact filling of the root canal by optimum dental material under aseptic conditions. Most pulp and periradicular pathologies are best controlled by nonsurgical techniques. Certain microorganisms exceptionally survive in root canals of the teeth that have become re-infected after previous root canal treatment. Almost all endodontic failures occur due to movement of bacteria into the root canals and periapical tissues. Hence, to prevent the growth of bacteria the cements should have antimicrobial property in addition to sealing ability.[3] Hence, the healing process and treatment outcome depends greatly upon the cardinal antimicrobial efficacy of the endodontic dental materials.

Mineral Trioxide Aggregate (MTA), class of calcium silicate cement was introduced by Torabinejad M, in 1993 and has been studied extensively since then. In teeth with open apex and non-vital teeth, MTA can be used due to its sealing ability and biocompatibility in place of calcium hydroxide cement. MTA also sets in the presence of moisture and blood. There are some drawbacks of this material such as slow setting kinetics, dentin discoloration, complicated handling properties. ${ }^{[4]}$

With the unceasing need for more new dental materials 'bio-ceramic' cements got introduced in dentistry. Biodentine (Septodont) which is termed as 'dentin in a capsule' due to its high physical and mechanical properties simulating tooth's dentin. It has ease of handling with excellent biocompatibility and bioactive nature. Biodentine has faster setting time, also metal impurities are eliminated so it does not cause dentin discoloration, an advantage over MTA.[5]

EndoSequence root repair material (RRM) introduced by Brasseler, is a new generation bioceramic material indicated for apical plugs in immature teeth. It is easy to handle due to its bioactive property and biocompatibility. When these bioceramic cements come in contact with the phosphate of tooth, hydroxyapatite crystals are deposited along their border. Thus, they show ability to induce tissue regeneration by their osteoinductive and osteoconductive properties. The aim of this study was to evaluate the antibacterial property of MTA, Biodentine and EndoSequence on Enterococcus faecalis.[6]

We wanted to compare and evaluate the antimicrobial property of Mineral Trioxide Aggregate (MTA), Biodentine, EndoSequence against the bacterial strains of Enterococcus faecalis using agar well diffusion system.

\section{METHODS}

The agar was removed, and wells were made, and the manipulated cements were immediately transferred in the wells. The diameter of the growth inhibition zones around the material was measured after 24-, 48- and 72-hours incubation at $37^{\circ} \mathrm{C}$. To compare the antimicrobial efficacy of the three cements the data obtained was analysed using Analysis of variance test and Tukey-Kramer Multiple comparison test.

\section{Ethical Considerations}

This was an in vitro study conducted after due approval from ethical committee (protocol number 0219/2018-19).

\section{Sample Size Determination}

Formula

$n=\frac{\left(\mathrm{z}^{2} \alpha / 2 \mathrm{p} * \mathrm{q}\right)}{\mathrm{d}^{2}}$

where, $\mathrm{n}=$ no. of samples

$\mathrm{z}=$ statistics corresponding to level of confidence

$\mathrm{p}=$ expected prevalence

$\mathrm{d}=$ precision

$\mathrm{q}=$ calculation of prevalence

$\mathrm{p}=0.90, \mathrm{~d}=5 \%$

$\alpha=0.05, \mathrm{z}_{\alpha / 2}=1.64$

Hence, the minimum amount of petri plates required is 10 according to the values obtained from the formula. Therefore, total sample size equals to 10 petri plates.

In this study, the test materials MTA, Biodentine and EndoSequence were manipulated to form their pellets. Agar diffusion method was used to compare and know the antimicrobial property of the three cements against Enterococcus faecalis (ATCC 29212). Diluted bacterial suspension was obtained of $5 \times 10^{6}$ colony forming units per $\mathrm{ml}$, in sterile Trypticase Soy Broth (TSB) by Spectrophotometer. Strains of the bacteria were confirmed by colony forming units and growth pattern. In petri plates base layer was formed of $10 \mathrm{ml}$ of Muller Hinton Agar. After the solidification of base layer, second layer was poured over it of $10 \mathrm{ml}$ of Mueller Hinton agar and $200 \mu \mathrm{L}$ of standardised microbial suspension. Three wells of $4 \mathrm{~mm}$ diameter and $4 \mathrm{~mm}$ deep were made after the solidification of second layer with a core borer in equidistant points and filled with freshly mixed cements.

Pre-diffusion of the cements was done for two hours at room temperature and then kept for incubation at $37^{\circ} \mathrm{C}$ and evaluated at 24, 48 and 72 hours. Diameter of zone of inhibition was measured with a $0.5 \mathrm{~mm}$ precision ruler and the results were expressed as the mean and standard deviation.

\section{Statistical Analysis}

The antimicrobial property of MTA, Biodentine, and EndoSequence was compared statistically using Analysis of variance test.

\section{RESULTS}

Mean inhibition zones of EndoSequence were largest at 24, 48 and 72 hours compared to that of Biodentine and MTA (Table 1). The results of Analysis of variance test suggested that the antimicrobial action of EndoSequence on Enterococcus faecalis was superior to that of Biodentine $(6.0 \mathrm{~mm})$ and MTA 
(5.6 mm), showing a mean inhibition zone of $11 \mathrm{~mm}$ after 72 hours. This difference was statistically significant against Biodentine and MTA $(\mathrm{p}<0.05)$ (Table 2).

\begin{tabular}{|c|c|c|c|c|c|c|}
\hline $\begin{array}{c}\text { Tested } \\
\text { Material }\end{array}$ & $\begin{array}{c}\text { Mean } \\
(24 \\
\text { Hrs.) } \\
(\mathrm{mm})\end{array}$ & $\begin{array}{c}\text { S.D. } \\
(24 \\
\text { Hrs.) }\end{array}$ & $\begin{array}{c}\text { Mean } \\
\text { (48 Hrs.) } \\
(\mathrm{mm})\end{array}$ & $\begin{array}{c}\text { S.D. } \\
(48 \\
\text { Hrs.) }\end{array}$ & $\begin{array}{c}\text { Mean } \\
\text { (72 } \\
\text { Hrs.) } \\
\text { (mm) }\end{array}$ & $\begin{array}{c}\text { S.D. } \\
\text { (72 } \\
\text { Hrs.) }\end{array}$ \\
\hline MTA & 5.0 & 0955 & 56 & 0.96 & 5.6 & 0.96 \\
\hline Biodentine & 5.5 & 0.966 & 6.0 & 0.98 & 6.0 & 0.98 \\
\hline EndoSequence & 10.6 & 2.591 & 11 & 2.593 & 11 & 2.593 \\
\hline \multicolumn{7}{|c|}{$\begin{array}{l}\text { Table 1. Mean Inhibition Zones of MTA, Biodentine and } \\
\text { EndoSequence after 24, } 48 \text { and } 72 \text { Hours (Measured in } \mathrm{mm} \text { ) }\end{array}$} \\
\hline
\end{tabular}

\begin{tabular}{|cccc|}
\hline Comparison & Mean Difference & q Value & P Value \\
MTA vs EndoSequence & -0.9620 & 14.050 & $\mathrm{p}<0.001$ \\
MTA vs Biodentine & 0.000 & 0.000 & $\mathrm{p}>0.05$ \\
Biodentine vs & 0.9620 & 14.050 & $\mathrm{p}<0.001$ \\
EndoSequence & Table 2. Repeated Measures ANOVA and Tukey-Kramer Multiple \\
Comparisons Test to Compare Antimicrobial Efficacy of MTA, \\
Biodentine and EndoSequence
\end{tabular}

\section{DISCUSSION}

The antimicrobial activity of MTA, Biodentine, and EndoSequence were evaluated. Enterococcus faecalis is found predominantly in progression of pulpal diseases and in endodontic failure. It is resistant to antimicrobial treatment and can survive in root canals for prolonged duration. Thus, it can be used for antimicrobial testing. [7] The most widely used method to compare the antimicrobial property is agar diffusion method. Many antimicrobial studies have employed this technique, but the zones of inhibition around the cements may vary due to difference in agar medium, bacterial strains, diffusion capacity of cements. ${ }^{[8]}$

The study showed that the diameter of the zone of inhibition varied between MTA, Biodentine and EndoSequence. The results showed that EndoSequence had largest zone of inhibition ( $11 \mathrm{~mm}$ at 72 hours) than Biodentine ( $6 \mathrm{~mm}$ at 72 hours) and MTA (5.6 mm at 72 hours). Results thus justify the highest antimicrobial efficacy of EndoSequence than Biodentine and MTA. Antimicrobial efficacy of Biodentine and MTA failed to show any statistical significance. This study suggests that EndoSequence is more potent in inhibiting the growth of bacteria than Biodentine and MTA.

MTA was introduced with the principle intention to be used as a root-end filling material due to their biocompatible nature. There are certain drawbacks of MTA as difficulty in handling, very slow setting reaction which eventually leads to microbial leakage, surface disintegration, loss of marginal adaptation. ${ }^{[9]}$ In order to overcome these difficulties novel bioceramic cements like Biodentine (Septodont) and EndoSequence (Brasseler) were introduced.

Biodentine shares both its indications with MTA, but does not have its disadvantages. Compared to MTA, Biodentine has ease of handling and it requires much less time for its final setting.[10]The greatest antimicrobial efficacy shown by EndoSequence can be attributed to some of its unique properties as justified by Shokouhinejad N. et al who states that the advantages of EndoSequence over other materials lies in its good biocompatible nature, high $\mathrm{pH}$ more than 12.5 , high resistance to washout, absence of shrinkage during setting.[11]
In literature there are studies showing equivalency in antimicrobial potential of MTA and EndoSequence. Lovato KF et al compared the antibacterial activity of EndoSequence and ProRoot MTA against clinical isolates of Enterococcus faecalis and they concluded that the material EndoSequence in putty and syringeble form and MTA had similar antibacterial efficacy against clinical strains of Enterococcus faecalis.[6] Even though MTA is the gold standard material, its disadvantages makes it technique sensitive in terms of clinical use. On the other hand, EndoSequence with excellent ease of handling becomes the strongest and better alternative over MTA and Biodentine that has to be considered.

\section{CONCLUSIONS}

All the three cements showed antimicrobial activity against Enterococcus faecalis (ATCC 29212). Compared to Biodentine and MTA, EndoSequence showed the largest zone of inhibition. Thus, EndoSequence is more potent in inhibiting the growth of bacteria than Biodentine and MTA.

\section{REFERENCES}

[1] Fouad AF, Zerella J, Barry J, et al. Molecular detection of Enterococcus species in root canals of therapy-resistant endodontic infections. Oral Surgery, Oral Medicine, Oral Pathology, Oral Radiology and Endodontology 2005;99(1):112-8.

[2] Zarrabi MH, Javidi M, Naderinasab M, et al. Comparative evaluation of antimicrobial activity of three cements: new endodontic cement (NEC), mineral trioxide aggregate (MTA) and Portland. Journal of Oral Science 2009;51(3):437-42.

[3] Priyalakshmi S, Ranjan M. Review on Biodentine-a bioactive dentin substitute. J Dent Med Sci 2014;13(1):137.

[4] Allazzam SM, Alamoudi NM, El Meligy OA. Clinical applications of Biodentine in pediatric dentistry: a review of literature. Journal of Oral Hygiene \& Health 2015;3:179.

[5] Lovato KF, Sedgley CM. Antibacterial activity of endosequence root repair material and proroot MTA against clinical isolates of Enterococcus faecalis. Journal of Endodontics 2011;37(11):1542-6.

[6] Heling I, Chandler NP. Antimicrobial effect of irrigant combinations within dentinal tubules. International Endodontic Journal 1998;31(1):8-14.

[7] Torabinejad M, Hong CU, Ford TRP, et al. Antibacterial effects of some root end filling materials. Journal of Endodontics 1995;21(8):403-6.

[8] Girish CS, Ponnappa KC, Girish TN, et al. Sealing ability of mineral trioxide aggregate, calcium phosphate and polymethylmethacrylate bone cements on root ends prepared using an Erbium: Yttriumaluminium garnet laser and ultrasonics evaluated by confocal laser scanning microscopy. Journal of Conservative Dentistry 2013;16(4):304-8. 
[9] Dalmia S, Gaikwad A, Samuel R, et al. antimicrobial efficacy of different endodontic sealers against enterococcus faecalis: an in vitro study. Journal of International Society of Preventive \& Community Dentistry 2018;8(2):104-9.

[10] Shokouhinejad N, Nekoofar MH, Ashoftehyazdi K, et al. Marginal adaptation of new bioceramic materials and mineral trioxide aggregate: a scanning electron microscopy study. Iranian Endodontic Journal 2014;9(2):144-8.

[11] Antunes HS, Gominho LF, Andrade-Junior CV, et al. Sealing ability of two root end filling materials in a bacterial nutrient leakage model. International Endodontic Journal 2016;49(10):960-5. 